\title{
SOME POWER OF AN ELEMENT IN A GARSIDE GROUP IS CONJUGATE TO A PERIODICALLY GEODESIC ELEMENT
}

\author{
EON-KYUNG LEE AND SANG-JIN LEE
}

\begin{abstract}
We show that for each element $g$ of a Garside group, there exists a positive integer $m$ such that $g^{m}$ is conjugate to a periodically geodesic element $h$, an element with $\left|h^{n}\right|_{\mathcal{D}}=|n| \cdot|h|_{\mathcal{D}}$ for all integers $n$, where $|g|_{\mathcal{D}}$ denotes the shortest word length of $g$ with respect to the set $\mathcal{D}$ of simple elements. We also show that there is a finite-time algorithm that computes, given an element of a Garside group, its stable super summit set.
\end{abstract}

2000 Mathematics Subject Classifications : 20F36, 20F10.

\section{INTRODUCTION}

For a finitely generated group $G$ and a finite set $X$ of semigroup generators for $G$, the translation number with respect to $X$ of an element $g \in G$ is defined by

$$
t_{G, X}(g)=\lim _{n \rightarrow \infty} \frac{\left|g^{n}\right|_{X}}{n},
$$

where $|\cdot|_{X}$ denotes the shortest word length in the alphabet $X$. If there is no confusion about the group $G$, we simply write $t_{X}(g)$ instead of $t_{G, X}(g)$. When $A$ is a group generator, $|g|_{A}$ and $t_{A}(g)$ indicate $|g|_{A \cup A^{-1}}$ and $t_{A \cup A^{-1}}(g)$, respectively. The following list is a part of the previous works on the discreteness properties of translation numbers in geometric and combinatorial groups: 14, 13, 21, 15, 1, 6, 5, 18, 17. An element $g$ is said to be periodically geodesic with respect to $X$ if $\left|g^{n}\right|_{X}=|n| \cdot|g|_{X}$ for all integers $n$.

It is well-known that, in a word hyperbolic group, all translation numbers are rational with uniformly bounded denominators. This follows from a claim of Gromov [14] and was accurately proved by Swenson [21]. In fact, Swenson proved that for every element $g$ of a word hyperbolic group, there exists a positive integer $m$ such that $g^{m}$ is conjugate to a periodically geodesic element $h$. (Moreover, the smallest such integer $m$ is uniformly bounded.) The rationality of the translation numbers in a word hyperbolic group is an immediate consequence of this result because the translation numbers are constant on the conjugacy class and $t_{X}\left(h^{n}\right)=|n| t_{X}(h)$ for all elements $h$ and integers $n$.

Before stating our results, we briefly review Garside groups. The class of Garside groups, first introduced by Dehornoy and Paris [8], provides a lattice-theoretic generalization of braid groups and Artin groups of finite type. Garside groups are equipped with a special element $\Delta$, called the Garside element, and a finite set $\mathcal{D}$, called the set of simple elements. Elements in a Garside group admit a unique normal form

$$
\Delta^{r} s_{1} \cdots s_{k},
$$

where $r \in \mathbb{Z}$ and $s_{1}, \ldots, s_{k} \in \mathcal{D} \backslash\{1, \Delta\}$. There are integer-valued invariants inf and sup for elements in a Garside group such that if $\Delta^{r} s_{1} \cdots s_{k}$ is the normal form of $g$, then $\inf (g)=r$ and $\sup (g)=r+k$. For an element $g \in G$, let $[g]$ denote its conjugacy class, that is, $[g]=\left\{h^{-1} g h\right.$ :

Date: September 10, 2018.

This work was supported by the Korea Research Foundation Grant funded by the Korean Government (MOEHRD, Basic Research Promotion Fund) (KRF-2006-312-C00469). 
$h \in G\}$. The conjugacy invariants $\inf _{s}$ and $\sup _{s}$ are defined as $\inf _{s}(g)=\max \{\inf (h): h \in[g]\}$ and $\sup _{s}(g)=\min \{\sup (h): h \in[g]\}$. The super summit set of $g$ is defined as

$$
[g]^{S}=\left\{h \in[g]: \inf (h)=\inf _{s}(g), \sup (h)=\sup _{s}(g)\right\} .
$$

Intuitively, the super summit set is the set of all elements in the conjugacy class that have the shortest normal form in that class. (Note that the number of simple elements $s_{i}$ in the normal form of $g$ is $\sup (g)-\inf (g)$, which decreases as $\inf (g)$ increases and as $\sup (g)$ decreases.)

We now state the main result of this note. See the next section for the definition of $\|\cdot\|$.

Theorem A (Theorem 4.3) Let $G$ be a Garside group with Garside element $\Delta$ and the set $\mathcal{D}$ of simple elements. Let $\|\Delta\|=N$ and $g \in G$. There exists a positive integer $n \leqslant N^{2}$ such that every element of the super summit set of $g^{n}$ is periodically geodesic with respect to $\mathcal{D}$.

We remark that our approach in Garside groups is different from Swenson's in word hyperbolic groups [21]. Swenson showed the existence of periodically geodesic power up to conjugacy, which implies the rationality of translation numbers. For Garside groups, we use the rationality of translation numbers, which is the main result of [17, in order to prove Theorem A, the existence of periodically geodesic power up to conjugacy. It seems very difficult to prove Theorem A without using the rationality of translation numbers. Therefore, the two results, the rationality of translation numbers and the existence of periodically geodesic powers, in word hyperbolic groups and in Garside groups are established in reverse order.

We explain our approach briefly. It is known that for an element $g$ of a Garside group, the shortest word length $|g|_{\mathcal{D}}$ is either $-\inf (g), \sup (g)$ or $\sup (g)-\inf (g)$. Because $\sup (g)=-\inf \left(g^{-1}\right)$, the proof of Theorem A can be reduced to proving that $g^{n}$ is conjugate to an inf-straight element for some $n \leqslant N$. (An element $h$ is inf-straight if $\inf \left(h^{m}\right)=m \inf (h)$ for all positive integers $m$.) This is a consequence of the results on the rationality of translation numbers in [17 and the inequality established in Theorem 3.2 ;

$$
\inf _{s}(g) \leqslant t_{\text {inf }}(g)<\inf _{s}(g)+1
$$

where $t_{\text {inf }}(g)=\lim _{n \rightarrow \infty} \inf \left(g^{n}\right) / n$.

Another interest of this note is to construct an algorithm for computing stable super summit sets in Garside groups. The stable super summit set of an element $g$ of a Garside group is defined as

$$
[g]^{\text {St }}=\left\{h \in[g]^{S}: h^{n} \in\left[g^{n}\right]^{S} \text { for all } n \geqslant 1\right\} .
$$

Namely, it is the set of all conjugates $h$ of $g$ whose every power $h^{n}$ has the shortest normal form in the conjugacy class of $g^{n}$. It is known that every stable super summit set is nonempty and satisfies most of the important properties of super summit sets [16]. However, as noted in [16], we need an affirmative answer to the following question in order to compute stable super summit sets.

Can we make a finite-time algorithm that decides, given an element $h$ in the conjugacy class of $g$, whether it belongs to the stable super summit set of $g$ ?

In this note, we solve the above problem, and hence obtain the following result.

Theorem B (Corollary 6.3). There is a finite-time algorithm that, given an element of a Garside group, computes its stable super summit set. 


\section{Garside groups}

We start with the definition of Garside groups and known solutions to the conjugacy problem in Garside groups. See [11, 3, 8, 7, 10, 19, 12] for details.

2.1. Garside monoids and groups. Let $M$ be a monoid. Let atoms be the elements $a \in M \backslash\{1\}$ such that $a=b c$ implies either $b=1$ or $c=1$. Let $\|a\|$ be the supremum of the lengths of all expressions of $a$ in terms of atoms. The monoid $M$ is said to be atomic if it is generated by its atoms and $\|a\|<\infty$ for any $a \in M$. In an atomic monoid $M$, there are partial orders $\leqslant_{L}$ and $\leqslant_{R}$ : $a \leqslant L b$ if $a c=b$ for some $c \in M ; a \leqslant R b$ if $c a=b$ for some $c \in M$.

Definition 2.1. An atomic monoid $M$ is called a Garside monoid if

(i) $M$ is finitely generated;

(ii) $M$ is left and right cancellative;

(iii) $\left(M, \leqslant_{L}\right)$ and $\left(M, \leqslant_{R}\right)$ are lattices;

(iv) there exists an element $\Delta$, called a Garside element, satisfying the following:

(a) for each $a \in M, a \leqslant_{L} \Delta$ if and only if $a \leqslant_{R} \Delta$;

(b) the set $\{a \in M: a \leqslant L \Delta\}$ generates $M$.

An element $a$ of $M$ is called a simple element if $a \leqslant_{L} \Delta$. Let $\mathcal{D}$ denote the set of all simple elements. Let $\wedge_{L}$ and $\vee_{L}$ denote the lattice operations of the poset $\left(M, \leqslant_{L}\right)$.

Garside monoids satisfy Ore's conditions, and thus embed in their groups of fractions. A Garside group is defined as the group of fractions of a Garside monoid. When $M$ is a Garside monoid and $G$ the group of fractions of $M$, we identify the elements of $M$ and their images in $G$ and call them positive elements of $G$. $M$ is called the positive monoid of $G$, often denoted by $G^{+}$.

The partial orders $\leqslant_{L}$ and $\leqslant_{R}$, and thus the lattice structures in the positive monoid $G^{+}$can be extended to the Garside group $G$ as follows: $g \leqslant_{L} h$ (respectively, $g \leqslant_{R} h$ ) for $g, h \in G$ if $g c=h$ (respectively, $c g=h$ ) for some $c \in G^{+}$.

For $g \in G$, there are integers $r \leqslant s$ such that $\Delta^{r} \leqslant L g \leqslant{ }_{L} \Delta^{s}$. Hence, the invariants $\inf (g)=$ $\max \left\{r \in \mathbb{Z}: \Delta^{r} \leqslant_{L} g\right\}, \sup (g)=\min \left\{s \in \mathbb{Z}: g \leqslant_{L} \Delta^{s}\right\}$ and $\operatorname{len}(g)=\sup (g)-\inf (g)$ are well-defined. It is known that, for $g \in G$, there exists a unique expression

$$
g=\Delta^{r} s_{1} \cdots s_{k}
$$

such that $s_{1}, \ldots, s_{k} \in \mathcal{D} \backslash\{1, \Delta\},\left(s_{i} s_{i+1} \cdots s_{k}\right) \wedge_{L} \Delta=s_{i}$ for $i=1, \ldots, k$, $\inf (g)=r$, and $\sup (g)=r+k$. Such an expression is called the normal form of $g$.

For $g \in G$, we denote its conjugacy class $\left\{h^{-1} g h: h \in G\right\}$ by $[g]$. Define $\inf _{s}(g)=\max \{\inf (h)$ : $h \in[g]\}$ and $\sup _{s}(g)=\min \{\sup (h): h \in[g]\}$. The super summit set $[g]^{S}$ and the stable super summit set $[g]^{\text {St }}$ are subsets of the conjugacy class of $g$ defined as follows:

$$
\begin{aligned}
{[g]^{S} } & =\left\{h \in[g]: \inf (h)=\inf _{s}(g) \text { and } \sup (h)=\sup _{s}(g)\right\} \\
{[g]^{\mathrm{St}} } & =\left\{h \in[g]^{S}: h^{k} \in\left[g^{k}\right]^{S} \text { for all positive integers } k\right\}
\end{aligned}
$$

It is well known that $[g]^{S}$ is finite and nonempty. Since $[g]^{\mathrm{St}}$ is a subset of $[g]^{S},[g]^{\mathrm{St}}$ is also a finite set. It is proved in [2, 16] that $[g]^{\mathrm{St}}$ is nonempty.

In the rest of the paper, if it is not specified, $G$ is assumed to be a Garside group, whose positive monoid is $G^{+}$, with Garside element $\Delta$ and the set $\mathcal{D}$ of simple elements, where $\|\Delta\|$ is simply written as $N$. 
2.2. Some results on inf and sup. Here, we collect some results on inf and sup. See [16 for Lemma 2.2 and Theorem 2.3, and [17] for the others.

For a positive element $a \in G^{+}$, define $\mathrm{L}^{\max }(a)=\Delta \wedge_{L} a$.

Lemma 2.2. Let $g \in G$ and $a \in G^{+}$. If $\inf (g a)>\inf (g)$, then $\inf \left(g \operatorname{L}^{\max }(a)\right)>\inf (g)$.

Theorem 2.3. Let $g \in G$. For all $n \geqslant 1$, the following hold.

(i) $n \inf _{s}(g) \leqslant \inf _{s}\left(g^{n}\right) \leqslant n \inf _{s}(g)+n-1$.

(ii) $n \sup _{s}(g)-(n-1) \leqslant \sup _{s}\left(g^{n}\right) \leqslant n \sup _{s}(g)$.

It is proved in [17, Lemmas 3.2 and 3.3] that the following limits are well-defined for all elements $g \in G$ :

$$
t_{\text {inf }}(g)=\lim _{n \rightarrow \infty} \frac{\inf \left(g^{n}\right)}{n} ; \quad t_{\text {sup }}(g)=\lim _{n \rightarrow \infty} \frac{\sup \left(g^{n}\right)}{n} ; \quad t_{\text {len }}(g)=\lim _{n \rightarrow \infty} \frac{\operatorname{len}\left(g^{n}\right)}{n} .
$$

Lemma 2.4. Let $g, h \in G$.

(i) $t_{\text {inf }}\left(h^{-1} g h\right)=t_{\text {inf }}(g)$ and $t_{\text {sup }}\left(h^{-1} g h\right)=t_{\text {sup }}(g)$.

(ii) For all $n \geqslant 1, t_{\mathrm{inf}}\left(g^{n}\right)=n t_{\mathrm{inf}}(g)$ and $t_{\mathrm{sup}}\left(g^{n}\right)=n t_{\mathrm{sup}}(g)$.

(iii) $\inf _{s}(g) \leqslant t_{\text {inf }}(g) \leqslant \inf _{s}(g)+1$ and $\sup _{s}(g)-1 \leqslant t_{\text {sup }}(g) \leqslant \sup _{s}(g)$.

(iv) Both $t_{\mathrm{inf}}(g)$ and $t_{\mathrm{sup}}(g)$ are rational numbers of the form $p / q$ for some integers $p$ and $q$ with $1 \leqslant q \leqslant N$.

For an element $g \in G$,

(i) $g$ is said to be inf-straight if $\inf (g)=t_{\text {inf }}(g)$;

(ii) $g$ is said to be sup-straight if $\sup (g)=t_{\text {sup }}(g)$.

Lemma 2.5. For every $g \in G$, the following conditions are equivalent.

(i) $g$ is inf-straight.

(ii) $\inf \left(g^{N}\right)=N \inf (g)$.

(iii) $\inf \left(g^{n}\right)=n \inf (g)$ for all $n \geqslant 1$.

Lemma 2.6. For every $g \in G$, the following conditions are equivalent.

(i) $g$ is sup-straight.

(ii) $\sup \left(g^{N}\right)=N \sup (g)$.

(iii) $\sup \left(g^{n}\right)=n \sup (g)$ for all $n \geqslant 1$.

Lemma 2.7. For every $g \in G$, the following conditions are equivalent.

(i) $g$ is conjugate to an inf-straight element.

(ii) $\inf _{s}(g)=t_{\text {inf }}(g)$.

(iii) $\inf _{s}\left(g^{N}\right)=N \inf _{s}(g)$.

(iv) $\inf _{s}\left(g^{k}\right)=k \inf _{s}(g)$ for all $k \geqslant 1$.

(v) For all $h \in[g]^{S}, h$ is inf-straight.

Lemma 2.8. For every $g \in G$, the following conditions are equivalent.

(i) $g$ is conjugate to a sup-straight element.

(ii) $\sup _{s}(g)=t_{\text {sup }}(g)$.

(iii) $\sup _{s}\left(g^{N}\right)=N \sup _{s}(g)$.

(iv) $\sup _{s}\left(g^{k}\right)=k \sup _{s}(g)$ for all $k \geqslant 1$.

(v) For all $h \in[g]^{S}, h$ is sup-straight. 


\section{Asymptotic Limit of inf}

For a real number $x$, let $\lfloor x\rfloor$ denote the largest integer less than or equal to $x$. From Lemma 2.4(iii), $\inf _{s}(g) \leqslant t_{\text {inf }}(g) \leqslant \inf _{s}(g)+1$ for all elements $g$ of a Garside group. The goal of this section is to show that $t_{\text {inf }}(g)=\inf _{s}(g)+1$ cannot happen, hence $\inf _{s}(g)=\left\lfloor t_{\text {inf }}(g)\right\rfloor$.

We first recall Schur's theorem. Schur 20] proved that for every positive integer $M$, there exists a positive integer $L$ such that for every partition of the set $\{1,2, \ldots, L\}$ into $M$ subsets, one of the subsets contains two numbers $n$ and $m$ together with their sum $n+m$. The smallest such integer $L$ is called the Schur number. As a corollary, we have the following lemma.

Lemma 3.1. Let $\mathbb{N}$ be the set of all positive integers. Let $T_{1}, \ldots, T_{M}$ be a finite collection of subsets of $\mathbb{N}$ such that $\mathbb{N}=T_{1} \cup \cdots \cup T_{M}$. Then there is at least one set $T_{k}$ that contains $n, m$ and $n+m$ for some $n, m \in \mathbb{N}$.

Theorem 3.2. For every element $g$ of a Garside group $G$, $\inf _{s}(g) \leqslant t_{\text {inf }}(g)<\inf _{s}(g)+1$.

Proof. Because $\inf _{s}(g) \leqslant t_{\text {inf }}(g) \leqslant \inf _{s}(g)+1$ by Lemma $2.4\left(\right.$ iii), it suffices to show that $t_{\text {inf }}(g) \neq$ $\inf _{s}(g)+1$. On the contrary, assume

$$
t_{\text {inf }}(g)=\inf _{s}(g)+1 .
$$

Since both $t_{\mathrm{inf}}$ and inf s $_{s}$ are conjugacy invariants, we may assume that $g$ belongs to its stable super summit set, and hence

$$
\inf \left(g^{n}\right)=\inf _{s}\left(g^{n}\right) \text { and } \sup \left(g^{n}\right)=\sup _{s}\left(g^{n}\right) \text { for all } n \geqslant 1 .
$$

Claim 3.3. For all $n, m \geqslant 1$, the following hold:

(i) $\inf \left(g^{n}\right)=n \inf (g)+n-1$;

(ii) $\inf \left(g^{n+m}\right)=\inf \left(g^{n}\right)+\inf \left(g^{m}\right)+1$;

(iii) $\operatorname{len}\left(g^{n}\right) \geqslant 1$.

Proof of Claim 3.3. Recall that $\inf \left(g^{n}\right)=\inf _{s}\left(g^{n}\right)$ and $\sup \left(g^{n}\right)=\sup _{s}\left(g^{n}\right)$ for all $n \geqslant 1$, because we have assumed that $g$ belongs to its stable super summit set.

(i) By Theorem $2.3(\mathrm{i}), \inf _{s}\left(g^{n}\right) \leqslant n \inf _{s}(g)+n-1$ for all $n \geqslant 1$. Assume that $\inf _{s}\left(g^{n}\right) \leqslant$ $n \inf _{s}(g)+n-2$ for some $n \geqslant 1$. By Lemma 2.4 (iii), $t_{\text {inf }}\left(g^{n}\right) \leqslant \inf _{s}\left(g^{n}\right)+1 \leqslant n \inf _{s}(g)+n-1$, and thus

$$
t_{\text {inf }}(g)=\frac{t_{\text {inf }}\left(g^{n}\right)}{n} \leqslant \inf _{s}(g)+1-\frac{1}{n}<\inf _{s}(g)+1 .
$$

This contradicts the assumption (11) which states $t_{\text {inf }}(g)=\inf _{s}(g)+1$.

(ii) By (i), $\inf _{s}\left(g^{n}\right)+\inf _{s}\left(g^{m}\right)+1=\left(n \inf _{s}(g)+n-1\right)+\left(m \inf _{s}(g)+m-1\right)+1$

$$
=(n+m) \inf _{s}(g)+n+m-1=\inf _{s}\left(g^{n+m}\right) .
$$

(iii) If $\operatorname{len}_{s}\left(g^{n}\right)=0$ for some $n \geqslant 1$, then $g^{n}$ is conjugate to $\Delta^{k}$ for some integer $k$. Therefore $\inf _{s}\left(g^{2 n}\right)=2 k=2 \inf _{s}\left(g^{n}\right)$ is an even integer. On the other hand, $\inf _{s}\left(g^{2 n}\right)=2 n \inf _{s}(g)+2 n-1$ by (i), hence $\inf _{s}\left(g^{2 n}\right)$ is an odd integer. It is a contradiction.

For $n \geqslant 1$, let $r_{n}=\inf \left(g^{n}\right)$ and $s_{n}=\mathrm{L}^{\max }\left(g^{n} \Delta^{-r_{n}}\right)$. Then,

$$
g^{n}=s_{n} a_{n} \Delta^{r_{n}}
$$

for some $a_{n} \in G^{+} \backslash \Delta G^{+}$. By Claim 3.3 for all $n, m \geqslant 1, s_{n} \neq 1$ and

$$
r_{n+m}=r_{n}+r_{m}+1 \text {. }
$$

Claim 3.4. If $s_{n}=s_{n+m}$ for some $n, m \geqslant 1$, then $\inf \left(a_{n} \Delta^{r_{n}} s_{m}\right)=r_{n}+1$. 
Proof of Claim 3.4. Note that

$$
\left(s_{n} a_{n} \Delta^{r_{n}}\right)\left(s_{m} a_{m} \Delta^{r_{m}}\right)=g^{n} g^{m}=g^{n+m}=s_{n+m} a_{n+m} \Delta^{r_{n+m}} .
$$

Since $s_{n}=s_{n+m}$ and $r_{n+m}=r_{n}+r_{m}+1$, we obtain $a_{n} \Delta^{r_{n}} s_{m} a_{m}=a_{n+m} \Delta^{r_{n}+1}$. Therefore

$$
\inf \left(a_{n} \Delta^{r_{n}} s_{m} a_{m}\right)=r_{n}+1>\inf \left(a_{n} \Delta^{r_{n}}\right) .
$$

Since $s_{m}=\mathrm{L}^{\max }\left(s_{m} a_{m}\right), \inf \left(a_{n} \Delta^{r_{n}} s_{m}\right)>\inf \left(a_{n} \Delta^{r_{n}}\right)=r_{n}$ by Lemma 2.2. Therefore,

$$
\inf \left(a_{n} \Delta^{r_{n}} s_{m}\right) \geqslant r_{n}+1 \text {. }
$$

On the other hand, $\inf \left(a_{n} \Delta^{r_{n}} s_{m}\right) \leqslant \inf \left(a_{n} \Delta^{r_{n}}\right)+1=r_{n}+1$. As a consequence, we obtain $\inf \left(a_{n} \Delta^{r_{n}} s_{m}\right)=r_{n}+1$.

For each simple element $s \in \mathcal{D}$, let $T_{s}=\left\{n \in \mathbb{N}: s_{n}=s\right\}$. Note that $\mathbb{N}=\cup_{s \in \mathcal{D}} T_{s}$ and $\mathcal{D}$ is a finite set. By Lemma 3.1, there exists a subset $T_{s}$ that contains $n, m$ and $n+m$ for some $n, m \geqslant 1$. ( $n$ and $m$ may not be distinct.) By definition of $T_{s}$,

$$
s_{n}=s_{m}=s_{n+m} .
$$

Applying Claim 3.4 to $(n, n+m)$ and $(m, n+m)$, we obtain

$$
\inf \left(a_{n} \Delta^{r_{n}} s_{m}\right)=r_{n}+1 \text { and } \inf \left(a_{m} \Delta^{r_{m}} s_{n}\right)=r_{m}+1,
$$

from which

$$
\begin{aligned}
r_{n+m} & =\inf _{s}\left(g^{n+m}\right) \geqslant \inf \left(s_{n}^{-1} g^{n+m} s_{n}\right)=\inf \left(s_{n}^{-1} g^{n} g^{m} s_{n}\right) \\
& =\inf \left(s_{n}^{-1}\left(s_{n} a_{n} \Delta^{r_{n}}\right)\left(s_{m} a_{m} \Delta^{r_{m}}\right) s_{n}\right)=\inf \left(\left(a_{n} \Delta^{r_{n}} s_{m}\right)\left(a_{m} \Delta^{r_{m}} s_{n}\right)\right) \\
& \geqslant \inf \left(a_{n} \Delta^{r_{n}} s_{m}\right)+\inf \left(a_{m} \Delta^{r_{m}} s_{n}\right)=\left(r_{n}+1\right)+\left(r_{m}+1\right) \\
& =r_{n}+r_{m}+2 .
\end{aligned}
$$

This contradicts (2).

Corollary 3.5. Let $G$ be a Garside group with Garside element $\Delta$, and let $N=\|\Delta\|$. For every element $g \in G$, we have

(i) $\inf _{s}(g) \leqslant t_{\text {inf }}(g) \leqslant \inf _{s}(g)+1-1 / N$;

(ii) $\sup _{s}(g)-1+1 / N \leqslant t_{\text {sup }}(g) \leqslant \sup _{s}(g)$;

(iii) $\operatorname{len}_{s}(g)-2+2 / N \leqslant t_{\text {len }}(g) \leqslant \operatorname{len}_{s}(g)$.

Proof. (i) Since $t_{\text {inf }}(g)$ is of the form $p / q$ for some $p, q \in \mathbb{Z}$ with $1 \leqslant q \leqslant N$ by Lemma 2.4 (iv), and $\inf _{s}(g) \leqslant t_{\text {inf }}(g)<\inf _{s}(g)+1$ by Theorem 3.2 we have $\inf _{s}(g) \leqslant t_{\text {inf }}(g) \leqslant \inf _{s}(g)+1-1 / N$ as desired.

(ii) It follows from (i) because $\sup _{s}(g)=-\inf _{s}\left(g^{-1}\right)$.

(iii) It follows from (i) and (ii).

The following example shows that the upper bound $\inf _{s}(g)+1-1 / N$ of $t_{\text {inf }}(g)$ in Corollary[3.5(i) is optimal.

Example 3.6. For an integer $N \geqslant 2$, let

$$
G=\left\langle x, y \mid x^{N}=y^{N}\right\rangle .
$$

It is a Garside group with Garside element $\Delta=x^{N}=y^{N}$ [8, Example 4], and $\|\Delta\|=N$. Let $g=x^{N-1}$. Then $\inf \left(g^{k}\right)=\lfloor k(N-1) / N\rfloor$ for all $k \geqslant 1$, hence $t_{\text {inf }}(g)=1-1 / N$. Since $\inf _{s}(g)=0$, $t_{\text {inf }}(g)=\inf _{s}(g)+1-1 / N$.

From Corollary 3.5 and Lemmas 2.7 and 2.8 , we have the following. 
Corollary 3.7. Let $g$ be an element of a Garside group.

(i) $g$ is conjugate to an inf-straight element if and only if $t_{\inf }(g)$ is an integer.

(ii) $g$ is conjugate to a sup-straight element if and only if $t_{\text {sup }}(g)$ is an integer.

By Lemma 2.7 and Corollary 3.7 $t_{\text {inf }}(g)$ is an integer if and only if $\inf _{s}\left(g^{n}\right)=n \inf _{s}(g)$ for all $n \geqslant 1$, and the same is true for $t_{\text {sup }}(g)$ and $\sup _{s}(g)$. However, the following example shows that we cannot expect such a property for $t_{\mathrm{len}}(g)$.

Example 3.8. Consider the group

$$
G=H \times H, \quad \text { where } H=\left\langle x, y \mid x^{2 p}=y^{2 p}\right\rangle \text { for } p \geqslant 1 .
$$

As in Example 3.6, $H$ is a Garside group with Garside element $\Delta_{H}=x^{2 p}=y^{2 p}$. Because $G$ is a cartesian product of $H$, it is a Garside group with Garside element $\Delta=\left(\Delta_{H}, \Delta_{H}\right)$ by [18, Theorem 4.1]. Let $g=\left(x^{-p}, y^{p}\right)$. Then, for all $n \geqslant 1, g^{n}=\left(x^{-n p}, y^{n p}\right)$ and it is not difficult to show that

$$
\inf \left(g^{n}\right)=\inf _{s}\left(g^{n}\right)=\lfloor-n / 2\rfloor \quad \text { and } \sup \left(g^{n}\right)=\sup _{s}\left(g^{n}\right)=\lceil n / 2\rceil .
$$

Therefore $t_{\text {inf }}(g)=-1 / 2$ and $t_{\text {sup }}(g)=1 / 2$ and it follows that $t_{\text {len }}(g)=t_{\text {sup }}(g)-t_{\text {inf }}(g)=1$. In particular $t_{\text {len }}(g)$ is an integer. However, it is not true that $\operatorname{len}_{s}\left(g^{n}\right)=n \operatorname{len}_{s}(g)$ for all $n \geqslant 1$. $\left(\right.$ For example, $\operatorname{len}_{s}\left(g^{2}\right)=\operatorname{len}_{s}(g)=2$.)

\section{Periodically geodesic elements}

Definition 4.1. Let $G$ be a group and $X$ be a finite set of semigroup generators for $G$. An element $g \in G$ is said to be periodically geodesic with respect to $X$ if $\left|g^{n}\right|_{X}=|n| \cdot|g|_{X}$ for all $n \in \mathbb{Z}$.

Note that if $X$ is closed under inversion, then $|g|_{X}=\left|g^{-1}\right|_{X}$, hence $g$ is periodically geodesic if $\left|g^{n}\right|_{X}=n|g|_{X}$ for all $n \geqslant 1$.

Lemma 4.2. Let $G$ be a Garside group with the set $\mathcal{D}$ of simple elements, and let $g \in G$. Every element $h \in[g]^{S}$ is periodically geodesic with respect to $\mathcal{D}$ if one of the following conditions holds.

(i) $\inf _{s}(g) \geqslant 0$ and $t_{\text {sup }}(g)$ is an integer.

(ii) $\sup _{s}(g) \leqslant 0$ and $t_{\mathrm{inf}}(g)$ is an integer.

(iii) Both $t_{\mathrm{inf}}(g)$ and $t_{\mathrm{sup}}(g)$ are integers.

Proof. It is well known (see [4 for example) that for any element $g \in G$

$$
|g|_{\mathcal{D}}=\left\{\begin{aligned}
\sup (g) & \text { if } \inf (g) \geqslant 0 \\
-\inf (g) & \text { if } \sup (g) \leqslant 0 \\
\operatorname{len}(g) & \text { otherwise }
\end{aligned}\right.
$$

(i) Since $\inf _{s}(g) \geqslant 0, \inf \left(h^{n}\right) \geqslant n \inf (h)=n \inf _{s}(g) \geqslant 0$ for all $n \geqslant 1$, hence

$$
\left|h^{n}\right|_{\mathcal{D}}=\sup \left(h^{n}\right) \text { for all } n \geqslant 1 .
$$

Since $t_{\text {sup }}(g)$ is an integer, $g$ is conjugate to a sup-straight element by Corollary 3.7 (ii). Since $h \in[g]^{S}, h$ is sup-straight by Lemma 2.8. Thus, by Lemma 2.6

$$
\sup \left(h^{n}\right)=n \sup (h) \text { for all } n \geqslant 1 .
$$

Consequently, $\left|h^{n}\right|_{\mathcal{D}}=\sup \left(h^{n}\right)=n \sup (h)=n|h|_{\mathcal{D}}$ for all $n \geqslant 1$.

(ii) It can be proved similarly to (i). 
(iii) If $\inf _{s}(g) \geqslant 0$ or $\sup _{s}(g) \leqslant 0$, then $h$ is periodically geodesic by (i) and (ii). So, we may assume $\inf _{s}(g)<0<\sup _{s}(g)$. Then $\inf \left(h^{n}\right) \leqslant \inf _{s}\left(g^{n}\right)<0<\sup _{s}\left(g^{n}\right) \leqslant \sup \left(h^{n}\right)$ for all $n \geqslant 1$ by Theorem 2.3, hence

$$
\left|h^{n}\right|_{\mathcal{D}}=\operatorname{len}\left(h^{n}\right) \text { for all } n \geqslant 1 .
$$

Since both $t_{\text {inf }}(g)$ and $t_{\text {sup }}(g)$ are integers, $g$ is conjugate to an element which is inf-straight and to an element which is sup-straight, by Corollary 3.7 Since $h \in[g]^{S}, h$ is both inf- and sup-straight by Lemmas 2.7 and 2.8 . Then, by Lemmas 2.5 and 2.6

$$
\operatorname{len}\left(h^{n}\right)=n \operatorname{len}(h) \text { for all } n \geqslant 1 .
$$

Consequently, $\left|h^{n}\right|_{\mathcal{D}}=\operatorname{len}\left(h^{n}\right)=n \operatorname{len}(h)=n|h|_{\mathcal{D}}$ for all $n \geqslant 1$.

We now establish the main theorem of this note.

Theorem 4.3. Let $G$ be a Garside group with Garside element $\Delta$ and the set $\mathcal{D}$ of simple elements. Let $\|\Delta\|=N$ and $g \in G$. There exists a positive integer $n \leqslant N^{2}$ such that every element of the super summit set of $g^{n}$ is periodically geodesic with respect to $\mathcal{D}$.

Proof. Let $t_{\mathrm{inf}}(g)=p_{1} / q_{1}$ and $t_{\mathrm{sup}}(g)=p_{2} / q_{2}$, where $p_{1}, p_{2}, q_{1}, q_{2} \in \mathbb{Z}$ and $1 \leqslant q_{1}, q_{2} \leqslant N$. Let $n$ be the least common multiple of $q_{1}$ and $q_{2}$. Then, $1 \leqslant n \leqslant N^{2}$. Since both $t_{\text {inf }}\left(g^{n}\right)=n p_{1} / q_{1}$ and $t_{\text {sup }}\left(g^{n}\right)=n p_{2} / q_{2}$ are integers, every element of the super summit set of $g^{n}$ is periodically geodesic with respect to $\mathcal{D}$ by Lemma 4.2

We remark that we can construct a finite-time algorithm that, given $g \in G$, computes the power $n$ in the above theorem by Lemma 4.2 and by using the algorithms for $t_{\text {inf }}(\cdot)$ described in 95 (Applying those algorithms for $t_{\text {inf }}(\cdot)$, we can do the same task for $\left.t_{\text {sup }}(\cdot).\right)$

\section{Computation of $t_{\text {inf }}(\cdot)$}

In [17, Theorem 3.9 (iii)], there is a finite-time algorithm for computing $t_{\mathrm{inf}}(g)$ given an element $g$ of a Garside group. This algorithm exploits two facts:

(i) $t_{\text {inf }}(g)=p / q$ for some integers $p, q$ with $1 \leqslant q \leqslant N$.

(ii) $\inf _{s}\left(g^{n}\right) / n \leqslant t_{\text {inf }}(g) \leqslant\left(\inf _{s}\left(g^{n}\right)+1\right) / n$ for all $n \geqslant 1$.

Therefore, for any $n \geqslant N^{2}, t_{\text {inf }}(g)$ is the unique rational number of the form $p / q$ in the closed interval $\left[\inf _{s}\left(g^{n}\right) / n,\left(\inf _{s}\left(g^{n}\right)+1\right) / n\right]$, where $p, q \in \mathbb{Z}$ with $1 \leqslant q \leqslant N$. Applying Theorem [3.2, this section shows different methods of computing $t_{\text {inf }}(\cdot)$.

Theorem 5.1. Let $G$ be a Garside group with Garside element $\Delta$, and let $N=\|\Delta\|$. For every $g \in G$, the following hold.

(i) $t_{\text {inf }}(g)=\max \left\{\inf _{s}\left(g^{k}\right) / k: k=1, \ldots, N\right\}$.

(ii) Let $1 \leqslant q \leqslant N$. $t_{\text {inf }}(g)=p / q$ for some $p \in \mathbb{Z}$ if and only if $\inf _{s}\left(g^{q N}\right)=N \inf _{s}\left(g^{q}\right)$. Furthermore, $p=\inf _{s}\left(g^{q}\right)$.

Proof. (i) Since $t_{\text {inf }}(g)=t_{\text {inf }}\left(g^{k}\right) / k \geqslant \inf _{s}\left(g^{k}\right) / k$ for all $k \geqslant 1$, it suffices to show that there exists $k \in\{1, \ldots, N\}$ such that $t_{\text {inf }}(g)=\inf _{s}\left(g^{k}\right) / k$. By Lemma 2.4 (iv), $t_{\text {inf }}(g)=p / q$ for some integers $p, q$ with $1 \leqslant q \leqslant N$. By Theorem 3.2 $\inf _{s}\left(g^{q}\right)=\left\lfloor t_{\text {inf }}\left(g^{q}\right)\right\rfloor=\left\lfloor q t_{\text {inf }}(g)\right\rfloor=p$, thus

$$
\inf _{s}\left(g^{q}\right) / q=p / q=t_{\text {inf }}(g) \text {. }
$$

(ii) Suppose that $\inf _{s}\left(g^{q N}\right)=N \inf _{s}\left(g^{q}\right)$. Then, $\inf _{s}\left(g^{q}\right)=t_{\text {inf }}\left(g^{q}\right)=q t_{\text {inf }}(g)$ by Lemma 2.7

Conversely, suppose that $t_{\text {inf }}(g)=p / q$ for some integer $p$. Then $t_{\text {inf }}\left(g^{q}\right)=q t_{\text {inf }}(g)=p$ is an integer, and it follows that $g^{q}$ is conjugate to an inf-straight element by Corollary 3.7 (i). This means that $\inf _{s}\left(g^{q N}\right)=N \inf _{s}\left(g^{q}\right)$ by Lemma 2.7. 
From Theorem 5.1 we obtain two ways to compute $t_{\text {inf }}(g)$.

(i) Compute $\inf _{s}\left(g^{k}\right)$ for $k=1, \ldots, N$, and then compute the maximum of $\left\{\inf _{s}\left(g^{k}\right) / k: k=\right.$ $1, \ldots, N\}$. Then, $t_{\text {inf }}(g)$ is this maximum value.

(ii) Compute $\inf _{s}\left(g^{k}\right)$ and $\inf _{s}\left(g^{k N}\right)$ for $k=1, \ldots, N$, and find $1 \leqslant q \leqslant N$ with $\inf _{s}\left(g^{q N}\right)=$ $N \inf _{s}\left(g^{q}\right)$. Then, $t_{\text {inf }}(g)=\inf _{s}\left(g^{q}\right) / q$.

\section{Computation of Stable super summit sets}

Recall the definition of the stable super summit set $[g]^{\mathrm{St}}$ of $g \in G$ :

$$
[g]^{\mathrm{St}}=\left\{h \in[g]^{S}: h^{n} \in\left[g^{n}\right]^{S} \text { for all } n \geqslant 1\right\} .
$$

As noted in $\S 1$, in order to compute the stable super summit set $[g]^{\text {St }}$, we need a finite-time algorithm that decides, given an element $h$ in the conjugacy class of $g$, whether $h$ belongs to $[g]^{\mathrm{St}}$. The existence of such an algorithm is obvious for the super summit set and the ultra summit set. However, for the stable super summit set, a naive algorithm will test whether $h^{n} \in\left[g^{n}\right]^{S}$ for all $n \geqslant 1$. This kind of algorithm does not halt in finite time. We resolve this problem by using Theorem 3.2 .

Theorem 6.1. Let $G$ be a Garside group with Garside element $\Delta$. Let $N=\|\Delta\|$ and $g \in G$.

(i) If $h \in[g]$ satisfies $\inf \left(h^{n}\right)=\inf _{s}\left(g^{n}\right)$ for all $1 \leqslant n \leqslant N$, then $\inf \left(h^{n}\right)=\inf _{s}\left(g^{n}\right)$ for all $n \geqslant 1$.

(ii) If $h \in[g]$ satisfies $\sup \left(h^{n}\right)=\sup _{s}\left(g^{n}\right)$ for all $1 \leqslant n \leqslant N$, then $\sup \left(h^{n}\right)=\sup _{s}\left(g^{n}\right)$ for all $n \geqslant 1$.

(iii) $[g]^{\mathrm{St}}=\left\{h \in[g]^{S}: h^{n} \in\left[g^{n}\right]^{S}\right.$ for all $\left.1 \leqslant n \leqslant N\right\}$.

Proof. We prove only (i), because (ii) can be proved similarly and (iii) is a consequence of (i) and (ii). Recall that $t_{\text {inf }}(g)=p / q$ for some integers $p, q$ with $1 \leqslant q \leqslant N$. From Theorem 3.2 .

$$
\inf _{s}\left(g^{n}\right)=\left\lfloor t_{\text {inf }}\left(g^{n}\right)\right\rfloor=\left\lfloor n t_{\text {inf }}(g)\right\rfloor=\lfloor n p / q\rfloor \text { for all } n \geqslant 1 .
$$

Choose any integer $n \geqslant 1$. Then, there exist non-negative integers $k$ and $r$ with $r<q$ such that $n=k q+r$. Since $\inf \left(h^{i}\right)=\inf _{s}\left(g^{i}\right)$ for all $1 \leqslant i \leqslant N$ by the hypothesis,

$$
\begin{aligned}
\inf \left(h^{n}\right) & \geqslant k \inf \left(h^{q}\right)+\inf \left(h^{r}\right)=k \inf _{s}\left(g^{q}\right)+\inf _{s}\left(g^{r}\right)=k p+\lfloor r p / q\rfloor \\
& =\lfloor(k q+r) p / q\rfloor=\left\lfloor n t_{\inf }(g)\right\rfloor=\inf _{s}\left(g^{n}\right) .
\end{aligned}
$$

By the definition of $\inf _{s}, \inf \left(h^{n}\right) \leqslant \inf _{s}\left(g^{n}\right)$. Consequently, $\inf \left(h^{n}\right)=\inf _{s}\left(g^{n}\right)$. Since $n$ was arbitrarily chosen, $\inf \left(h^{n}\right)=\inf _{s}\left(g^{n}\right)$ for all $n \geqslant 1$.

From Theorem 6.1 (iii), it suffices to check if $h^{n} \in\left[g^{n}\right]^{S}$ only for all $1 \leqslant n \leqslant N$ in order to decide $h \in[g]^{\mathrm{St}}$.

Corollary 6.2. There is a finite-time algorithm that, given elements $g$ and $h$ of a Garside group, decides whether $h \in[g]^{\mathrm{St}}$ or not.

Using the algorithm in the above corollary, together with the results in [16], we can make a finite-time algorithm for computing the stable super summit set:

(i) Compute an element $h_{0}$ in $[g]^{\mathrm{St}}$.

By Corollary 3.12 in [16, there is a finite-time algorithm that, given $g \in G$ and $n \geqslant 1$, computes an element $h \in[g]$ with the property that $h^{k} \in\left[g^{k}\right]^{S}$ for all $k \in\{1, \ldots, n\}$. By Theorem 6.1 such an element $h$ belongs to $[g]^{\text {St }}$ if we take $n \geqslant N$. 
(ii) Compute all the elements in $[g]^{\mathrm{St}}$ from $h_{0} \in[g]^{\mathrm{St}}$ obtained in the above step. By Corollary 4.5 (iii) in [16], for any $h \in[g]^{\text {St }}$, there exists a finite sequence

$$
h_{0} \rightarrow h_{1} \rightarrow \cdots \rightarrow h_{m}=h
$$

such that for each $i=1, \ldots, m, h_{i} \in[g]^{\text {St }}$ and $h_{i}=s_{i}^{-1} h_{i-1} s_{i}$ for some simple element $s_{i}$. Therefore, using Corollary 6.2, the stable super summit set can be computed in the way to compute super summit sets: Define $V_{1}=\left\{h_{0}\right\}$, and then recursively compute $V_{i}=\left\{s^{-1} h s: s \in \mathcal{D}, h \in V_{i-1}\right\} \cap[g]^{\mathrm{St}}$; Then $V_{1} \subset V_{2} \subset \cdots \subset[g]^{\mathrm{St}}$; Since $[g]^{\mathrm{St}}$ is a finite set, there exists $k \geqslant 1$ such that $V_{k}=V_{k+1}$; Then $V_{k}=[g]^{\mathrm{St}}$.

Corollary 6.3. There is a finite-time algorithm that, given an element $g$ of a Garside group, computes $[g]^{\mathrm{St}}$.

\section{REFERENCES}

[1] M. Bestvina, 'Non-positively curved aspects of Artin groups of finite type', Geom. Topol. 3 (1999) 269-302.

[2] J. S. Birman, V. Gebhardt and J. González-Meneses, 'Conjugacy in Garside groups I: Cyclings, powers, and rigidity', Groups, Geom. Dyn. 1 (2007) 221-279.

[3] J. S. Birman, K. H. Ko and S. J. Lee, 'A new approach to the word and conjugacy problems in the braid groups', Adv. Math. 139 (1998) 322-353.

[4] R. Charney, 'Geodesic automation and growth functions for Artin groups of finite type', Math. Ann. 301 (1995) 307-324.

[5] R. Charney, J. Meier and K. Whittlesey, 'Bestvina's normal form complex and the homology of Garside groups', Geom. Dedicata 105 (2004) 171-188.

[6] G. R. Conner, 'Discreteness properties of translation numbers in solvable groups', J. Group Theory 3 (2000) 77-94.

[7] P. Dehornoy, 'Groupes de Garside', Ann. Sci. École. Norm. Sup. 35 (2002) 267-306.

[8] P. Dehornoy and L. Paris, 'Gaussian groups and Garside groups, two generalisations of Artin groups', Proc. London Math. Soc. (3) 79 (1999) 569-604.

[9] D. B. A. Epstein, J. W. Cannon, D. F. Holt, S. V. F. Levy, M. S. Paterson and W. P. Thurston, Word processing in groups, Chapter 9 (Jones and Bartlett Publishers, Boston, MA, 1992).

[10] N. Franco and J. González-Meneses, 'Conjugacy problem for braid groups and Garside groups', J. Algebra 266 (2003) 112-132.

[11] F. A. Garside, 'The braid group and other groups', Quart. J. Math. 20 (1969) 235-254.

[12] V. Gebhardt, 'A new approach to the conjugacy problem in Garside groups', J. Algebra 292 (2005) 282302.

[13] S. M. Gersten and H. B. Short, 'Rational subgroups of biautomatic groups', Ann. of Math. (2) 134 (1991) $125-158$.

[14] M. Gromov, 'Hyperbolic groups', Essays in group theory, 75-263 (Springer-Verlag, New York, 1987).

[15] I. Kapovich, 'Small cancellation groups and translation numbers', Trans. Amer. Math. Soc. 349 (1997) $1851-1875$.

[16] E.-K. Lee and S. J. Lee, 'Abelian subgroups of Garside groups', Comm. Algebra 36 (2008) 1121-1139.

[17] E.-K. Lee and S. J. Lee, 'Translation numbers in a Garside group are rational with uniformly bounded denominators', J. Pure Appl. Algebra 211 (2007) 732-743.

[18] S. J. Lee, 'Garside groups are strongly translation discrete', J. Algebra 309 (2007) 594-609.

[19] M. Picantin, 'The conjugacy problem in small Gaussian groups', Comm. Algebra 29 (2001) 1021-1039.

[20] I. Schur, 'Uber die Kongruenz $x^{m}+y^{m} \equiv z^{m}(\bmod p)$ ', Jber. Deutsch. Math.-Verin. 25 (1916) 114-116.

[21] E. Swenson, 'Hyperbolic elements in negatively curved groups', Geom. Dedicata 55 (1995) 199-210.

Department of Applied Mathematics, Sejong University, Seoul, 143-747, Korea

E-mail address: eonkyung@sejong.ac.kr

Department of Mathematics, Konkuk University, Seoul, 143-701, Korea

E-mail address: sangjin@konkuk.ac.kr 